

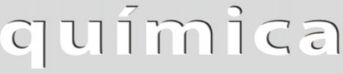

\section{A LIQUID CHROMATOGRAPHY METHOD FOR DETERMINATION OF PYRETHROIDS INSECTICIDES RESIDUES IN BEANS.}

\author{
Sérgio Henrique Monteiro ${ }^{(1)}$, Cláudia Helena Pastor Ciscato ${ }^{(1)}$, Amir Bertoni Gebara $^{(1)}$ e Jorge Cesar Masini ${ }^{(2)}$
}

\begin{abstract}
${ }^{(1)}$ Laboratório de Resíduos de Pesticidas, Instituto Biológico, Av. Conselheiro Rodrigues Alves, 1252, Vila Mariana, 04014-002 São Paulo - SP, Brasil. E-mail: monteiro@biologico.sp.gov.br, ciscato@biologico.sp.gov.br, gebara@biologico.sp.gov.br
\end{abstract}

${ }^{(2) I n s t i t u t o ~ d e ~ Q u i ́ m i c a, ~ U n i v e r s i d a d e ~ d e ~ S a ̃ o ~ P a u l o, ~ A v . ~ P r o f . ~ L i n e u ~ P r e s t e s, ~ 748, ~ B u t a n t a ̃, ~ 05508-000, ~ S a ̃ o ~ P a u l o ~-~ S P, ~ B r a s i l . ~ E-m a i l: ~}$ jcmasini@iq.usp.br

A rapid liquid chromatographic (LC) method was developed for simultaneous determination of 7 pyrethroid insecticides in beans. Residues were extracted from beans with acetone, followed by partition with ethyl acetate/cyclohexane $(1+1)$ and clean up by gel-permeation chromatography. LC separation was performed on a LiChrospher 100 RP-18 column using acetonitrile/water $(8+2)$ as mobile phase. The pesticides were detected by molecular absorption spectrophotometry at $212 \mathrm{~nm}$. Recoveries of 7 pyrethroids fortified at $0.010 ; 0.10 ; 1.0$ mg kg-1 levels were within the range 71-105\%. Advantageous features of this method are: the quantification limits, which were between 0.004 and $0.011 \mathrm{mg} \mathrm{kg}-1$, and the simplicity in the sample treatment, which requires less clean-up if compared with the GC-ECD determination. No residues of pyrethroids were detected in 48 bean samples commercialized in São Paulo City during 2008.

Key words: pesticide residue determination, high performance liquid chromatography, method development, method validation

\section{Introdução}

De acordo com Head, citado por Hirata [1], as piretrinas são extraídas de flores da Chrysanthemum Cinerariaefolium, muito semelhante a margarida comum; as propriedades destas plantas são conhecidas pelo menos desde o primeiro século da nossa era, como testemunhado pelos chineses, mas foram os comerciantes armênios que a trouxeram do Cáucaso e de regiões vizinhas, para a Europa Ocidental [2]. O piretro utilizado há aproximadamente 400 anos no controle de insetos, era conhecido como o "Pó da Pérsia". Em 1880 o piretro foi introduzido no Japão, que passou a exportá-lo em 1886, tendo sido, até a Primeira Guerra Mundial, o principal produtor [3].

Os resultados levantados nas avaliações de resíduos de pesticidas servem para dar suporte aos serviços governamentais de extensão agrícola, para aperfeiçoar o uso de pesticidas e práticas de aplicação por agricultores, bem como para minimizar os riscos ao consumidor [4].

Para dar suporte ao monitoramento de resíduos de pesticidas, novas técnicas e métodos são necessários para melhorar a qualidade da análise e também facilitar o trabalho dos analistas.

Hoje em dia muitos métodos modernos são propostos para esta finalidade, como os métodos descritos na revisão da literatura feita por Prestes et al. [5], porém, na maioria das vezes os laboratórios não possuem estrutura e tecnologia para utilizar esses métodos.

A cromatografia a líquido de alta eficiência (CLAE) é uma técnica alternativa para a detecção e quantificação de alguns pesticidas, em especial os piretróides que possuem alta massa molecular em comparação com a maioria dos pesticidas.

Usualmente os resíduos de inseticidas piretróides são determinados por cromatografia a gás com detector de captura de elétrons (CG-ECD) ou detector de espectrometria de massa (CG-MS), como citado por Vieira et al. [6], porém a determinação de piretróides por cromatografia a gás, principalmente quando colunas 
capilares são utilizadas, identificam vários isômeros que muitas vezes não são separados de forma satisfatória, apresentando baixa resolução cromatográfica. $\mathrm{Na}$ determinação por CLAE, utilizando colunas de fase reversa, esta dificuldade é descartada devido a observação de apenas um pico para cada piretróide, salvo para permetrina que apresenta seus isômeros cis e trans com ótima resolução.

Para uma otimização no emprego de CLAE e CG em análise de resíduos de pesticidas em alimentos (origem vegetal e animal) e amostras ambientais, é necessário que ocorra um preparo adequado da matriz a ser analisada utilizando técnicas apropriadas, como também, na extração, purificação, identificação e quantificação dos pesticidas. A CLAE permite a determinação de compostos não voláteis e termo-lábeis e, além disso, o método não requer a perfeita limpeza (clean-up) das amostras, como acontece no emprego do CG-ECD [7].

Segundo informações recolhidas na página eletrônica da EMBRAPA, o feijão é um alimento básico para o brasileiro, chegando a ser um componente obrigatório na dieta diária da população. A média atual de consumo de feijão é de $14,9 \mathrm{~kg} / \mathrm{brasileiro/ano.} \mathrm{A}$ preferência do consumidor é regionalizada e diferenciada principalmente quanto à cor e ao tipo de grão [8].

A validação de um método estabelece, através de estudos sistemáticos de laboratório, que o método é adequado à finalidade, isto é, suas características de desempenho são capazes de produzir resultados correspondentes às necessidades do problema analítico [9].

Os parâmetros de validação de métodos analíticos envolvem: Especificidade/Seletividade, Função de Resposta (curva analítica), Intervalo de Trabalho (Faixa), Linearidade, Exatidão, Precisão, Limite de Detecção e Limite de Quantificação [10].

No presente trabalho será descrito o desenvolvimento e a validação de um método de cromatografia de fase líquida, com detecção por espectrofotometria de absorção molecular na região do ultravioleta, para determinação de resíduos de piretróides em feijão.

\section{Material e Métodos}

\section{Reagentes e soluções}

Os reagentes utilizados no método foram: Sulfato de sódio PA (Quimex); Cloreto de sódio PA (Quimex); Acetona RP (Tedia); Acetato de etila RP (Tedia); Cicloexano RP (Tedia); Resina BIO-BEADS ${ }^{\circledR}$ SX3 (Bio-Rad); Acetonitrila HPLC (Tedia); Água grau I.

Os padrões primários dos produtos, fenvaler- ato $99 \%$ (Riedel-de-Haën), fempropatrina 97\% (Riedel-de-Haën), deltametrina 99,15\% (Ultra Scientific), lambda-cialotrina 99\% (Accustandard), cipermetrina 98\% (Ultra Scientific), bifentrina 99,7\% (Accustandard) e permetrina 99,6\% (Dr. Ehrenstorfer), foram inicialmente dissolvidos em acetonitrila, preparando-se soluções estoques de concentrações $1000 \mathrm{ng} \mu \mathrm{L}^{-1}$. As demais soluções foram preparadas em acetonitrila/água $(8+2)$.

\section{Extração}

Pesar em frasco reagente de $250 \mathrm{~mL}, 20 \mathrm{~g}$ de amostra previamente triturada e homogeneizada. Utilizar para isso uma balança analítica com resolução até $0,1 \mathrm{mg}$. Adicionar 36,5 g de água desionizada e homogeneizar agitando manualmente. Adicionar $100 \mathrm{~mL}$ de acetona. Homogeneizar no Ultra Turrax ${ }^{\circledR}$ por 3 minutos. Adicionar $17,5 \mathrm{~g}$ de $\mathrm{NaCl}$. Homogeneizar no Ultra Turrax ${ }^{\circledR}$ por mais 1 minuto.

\section{Partição}

Adicionar $50 \mathrm{~mL}$ de solução acetato de etila/ cicloexano $(1+1)$. Homogeneizar no Ultra Turrax ${ }^{\circledR}$ por 1 minuto. Deixar em repouso para separar as fases por aproximadamente 30 minutos. Coletar $100 \mathrm{~mL}$ da fase orgânica (sobrenadante). Filtrar em funil analítico com algodão coberto com $50 \mathrm{~g}$ de sulfato de sódio anidro, recolhendo o filtrado em balão de fundo redondo de 250 $\mathrm{mL}$. Lavar o sulfato de sódio duas vezes com $10 \mathrm{~mL}$ de solução acetato de etila/cicloexano (1+1). Concentrar no evaporador rotativo a $40^{\circ} \mathrm{C}$ e 40 psi até o volume de aproximadamente $2 \mathrm{~mL}$. Secar com fluxo suave de nitrogênio. Ressuspender em $10 \mathrm{~mL}$ da solução acetato de etila/cicloexano $(1+1)$.

\section{Purificação em coluna de cromatografia de permeação a gel (GPC)}

Filtrar os $10 \mathrm{~mL}$ obtidos anteriormente em $\mathrm{Na}_{2} \mathrm{SO}_{4}$ anidro com auxílio de funil pequeno de vidro e algodão lavado com diclorometano. Injetar $5 \mathrm{~mL}$, da solução filtrada, no GPC com vazão de $5 \mathrm{~mL} \mathrm{~min}{ }^{-1}$ usando a solução acetato de etila/cicloexano (1+1) como eluente. Desprezar a solução eluida referente aos primeiros 18 minutos $(90 \mathrm{~mL})$. Recolher em balão de $250 \mathrm{~mL}$ os próximos $110 \mathrm{~mL}$, onde se encontram os piretróides. Este volume corresponde a 22 minutos de tempo de eluição. Efetuar a limpeza do GPC eluindo a solução por mais 5 minutos $(25 \mathrm{~mL})$. Concentrar em evaporador rotativo, a $40^{\circ} \mathrm{C}$ e pressão de $55 \mathrm{psi}$, os $110 \mathrm{~mL}$ recolhidos no GPC até um volume de aproximadamente $2 \mathrm{~mL}$. Secar com fluxo 
suave de $\mathrm{N}_{2}$. Lavar o balão com $5 \mathrm{~mL}$ de acetonitrila/água $(8+2)$ e recolher em tubo graduado de $10 \mathrm{~mL}$ com rosca esmerilhada. Injetar $100 \mu \mathrm{L}$ no cromatógrafo a líquido com detector UV-Visível.

\section{Condições cromatográficas}

Cromatógrafo de fase líquida $\operatorname{Varian}^{\circledR}$ : Bomba 9012, Detector UV-Visível 9050; Coluna: Merck $^{\circledR}$ LiChrospher $^{\circledR}$ RP $185 \mu \mathrm{m}$ x 15 cm x 0,46 mm; Vazão:

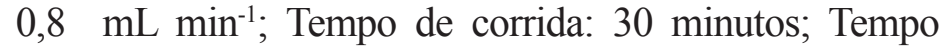
de estabilização: 2 minutos; Comprimento de onda: 212 nm; Volume de injeção: $100 \mu \mathrm{L}$; Fase móvel: acetonitrila/ água (8+2); Integrador $4400 \operatorname{Varian}^{\circledR}$; Atenuação: 16; Velocidade do papel: $0,5 \mathrm{~cm} \mathrm{~min}^{-1}$; Inibição de integração: integrar após 11 min; Tipo de integração: Valley-to-Valley Baselines.

\section{Validação}

Os parâmetros de validação estudados foram: Especificidade/Seletividade, Função de Resposta (curva analítica), Intervalo de Trabalho (Faixa), Linearidade, Exatidão, Precisão (Repetitividade e Reprodutibilidade), Limite de Detecção e Limite de Quantificação.

Para avaliação da Função de Resposta/Intervalo de Trabalho/Linearidade foram construídas curvas analíticas de calibração para cada piretróide com con-

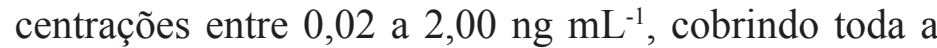
faixa de resposta obtida nos três níveis de fortificação estudados, obtidas a partir de 3 injeções consecutivas de 8 concentrações de soluções-padrão injetadas em ordem crescente de suas concentrações.

Limite de Detecção/Limite de Quantificação: O limite de detecção foi calculado como a razão sinal/ ruído com valores de 2:1 e o limite de quantificação foi estabelecido pela razão sinal/ruído 10:1, obtida por meio da comparação dos sinais medidos da amostra com baixas concentrações conhecidas do analito, com as do branco $[11,12,13,14]$.

Seletividade: Foram analisadas 7 repetições do branco (amostras testemunhas de feijão adquiridas com produtores orgânicos) para observar a não interferência de picos na região cromatográfica de interesse.

Além disso, uma amostra fortificada foi injetada em cromatógrafo a gás com detector de captura de elétrons, comparando-se os tempos de retenção com os tempos característicos dos padrões de piretróides injetados nas mesmas condições.

Exatidão/Precisão: As amostras testemunhas de feijão foram fortificadas em 3 níveis: 0,$010 ; 0,10$ e $1,0 \mathrm{mg} \mathrm{kg}^{-1}$ e realizadas 7 repetições para cada nível. Os valores de recuperação e coeficiente de variação foram comparados com os intervalos propostos por diferentes órgãos como Codex Alimentarius, SANCO, EPA e AOAC/FAO/IAEA/IUPAC.

\section{Amostras analisadas}

Foram analisadas 48 amostras de feijão adquiridas no comércio varejista da cidade de São Paulo durante o ano de 2008 e coletadas aleatoriamente. As amostras foram analisadas no mesmo dia em que deram entrada no laboratório.

\section{Resultados e Discussão}

\section{Cultura Estudada}

Os critérios para a escolha da cultura estudada seguiram dois princípios: as culturas mais sujeitas à aplicação de pesticidas piretróides e a maior porcentagem de comercialização destas culturas no país.

Desta forma foi escolhido o feijão para ser estudado, pois, além do grande consumo deste produto pelos brasileiros, ele também apresenta registro para a maioria dos piretróides estudados, como mostrado na Tabela 1.

Tabela 1 - Limites máximos de resíduo permitido dos piretróides estudados, na cultura de feijão [15].

\begin{tabular}{cccc}
\hline Riretróides & Modalidade de emprego & $\mathrm{LMR}^{*}\left(\mathrm{mg} \mathrm{kg}^{-1}\right)$ & Intervalo de segurança \\
\hline Permetrina & Sem registro & \\
Cipermetrina & foliar & 0,05 & 14 dias \\
Eenvalerate & & Sem registro & \\
Eenpropatrina & foliar & 0,01 & 14 dias \\
Deltametrina & foliar & 0,2 & 16 dias \\
& armazenade & 0,2 & 30 dias \\
Lambda-cialotrina & foliar & 0,05 & 15 dias \\
Bifentrina & foliar & 0,02 & 20 dias \\
\hline *Limite Máximo & & &
\end{tabular}

\section{Condições cromatográficas}

Foram realizados estudos para a verificação das melhores condições de análise, variando-se os seguintes parâmetros: fase estacionária, fase móvel, vazão da fase móvel e comprimento de onda.

Os comprimentos de onda utilizados para a 
determinação dos piretróides foram selecionados a partir dos espectros de absorção molecular na região do UV-Vis obtidos entre 190 e $400 \mathrm{~nm}$. O comprimento de onda de $212 \mathrm{~nm}$ apresentou melhor resultado, onde os piretróides apresentaram maior absortividade, com menor variação da linha de base cromatográfica.

Com injeções nas condições cromatográficas apresentadas em "Material e Métodos", observa-se as resoluções cromatográficas apresentadas na Tabela 2. A resolução foi maior que 1,5 para todos os picos e desta forma é possível à determinação quantitativa para todos os piretróides na mesma amostra.

Tabela 2 - Valores de resolução dos picos para solução padrão 0,02 ng $\mu \mathrm{L}-1$.

\begin{tabular}{cc}
\hline Riretróides & Resolução \\
\hline Eenpropatrina/Lambda-cialotrina & 1,6 \\
Lambda-cialotrina/Fenvalerate & 1,5 \\
Eenvalerato/Bifentrina & 8,5 \\
Cipermetrina/Deltametrina & 2,0 \\
Deltametrina/Cis-permetrina & 3,7 \\
Cis-permetrina/Trans-permetrina & 3,3 \\
\hline
\end{tabular}
amostra:

Metodologia de extração e purificação da

A técnica de extração utilizada foi a mesma descrita no método DFG-S19 [16, 17], com modificações [18], sendo utilizados apenas $100 \mathrm{~mL}$ de acetona em $20 \mathrm{~g}$ de amostra. Foi realizada uma partição utilizando apenas $50 \mathrm{~mL}$ de acetato de etila/ciclohexano $(1+1)$, seguindo-se a purificação por cromatografia de permeação em gel (GPC), também utilizada no método DFG-S19, com modificações.

\section{Validação do método}

Os piretróides apresentam vários isômeros, muitas vezes de difícil separação. A cromatografia líquida de fase reversa de modo geral não separa os isômeros, com exceção da permetrina, que apresentou os picos referentes a suas formas cis e trans. Essa característica facilita a interpretação dos cromatogramas, sem prejudicar a informação sobre presença ou não de um dado piretróide.

Observa-se na Tabela 3 que todos os piretróides apresentaram coeficientes de correlação > 0,997, indicando uma linearidade de resposta nas condições cromatográficas especificadas, o que demonstra a habilidade do método em obter os resultados de teste proporcionais a concentração dos piretróides estudados, na faixa de trabalho pretendida.

Tabela 3 - Dados da equação linear e coeficiente de correlação das curvas analíticas de calibração.

\begin{tabular}{cccccc}
\hline Piretróide & $\mathrm{a} 10^{-4}$ & $\delta$ de a $10^{-4}$ & $\mathrm{~b} 10^{-4}$ & $\delta$ de b $10^{-4}$ & $\mathrm{f}^{2}$ \\
\hline Bifentrina & -3 & 3 & 116 & 3 & 0,997 \\
Eenpropatrina & -2 & 1 & 57 & 1 & 0,997 \\
Eenvalerate & -2 & 1 & 74 & 1 & 0,998 \\
Cipermetrina & -2 & 1,7 & 96 & 2 & 0,998 \\
Deltametrina & 0,6 & 1 & 88 & 1 & 0,999 \\
Trans-Permetrina & 0,6 & 0,9 & 93 & 1 & 0,999 \\
Cis-Permetrina & $-0,6$ & 0,9 & 94 & 1 & 0,999 \\
Lambda-Cialotrina & 0,7 & 2 & 88 & 2 & 0,999 \\
\hline Nota: $\mathrm{y}=\mathrm{a}+\mathrm{bx}, \mathrm{a}=$ coeficiente linear, $\mathrm{b}=$ coeficiente angular. $\delta=$ desvio padrão, $\mathrm{r}^{2}=$ coeficiente de \\
correlação.
\end{tabular}

Como substâncias diferentes podem apresentar respostas similares em dadas condições deve-se proceder à verificação da especificidade, seguida por outras técnicas comprobatórias.

A seletividade foi verificada pela observação dos cromatogramas das amostras em branco, que não apresentaram nenhum pico na região cromatográfica de interesse. E para se ter certeza de que os picos encontrados nos estudos de fortificação são realmente os picos característicos dos piretróides, uma amostra em estudo foi injetada em cromatógrafo a gás com detector de captura de elétrons, comparando-se os tempos de retenção com os tempos característicos dos padrões de piretróides injetados nas mesmas condições. Isto confirmou a identificação qualitativa dos piretróides feitas por CLAE, comprovando sua seletividade.

Observa-se na Tabela 4, que todos os piretróides apresentaram recuperações dentro do intervalo de 70 a $120 \%$, proposto pela Agência de Proteção Ambiental dos Estados Unidos da América [19] e pelo Codex Alimentarius, [20]. Os valores estão também de acordo com a Comissão Européia para monitoramento de resíduos de pesticidas, que propõe um intervalo de 70 a $120 \%$ [21]. Todas as recuperações variaram entre 70 - 106\% (Tabela 4), de acordo com os intervalos aceitos 
para as concentrações estudadas; o método proposto mostra-se, assim, exato.

Tabela 4 - Recuperações, Limites de Detecção e Limites de Quantificação.

Tabela 4 - Recuperações, Limites de Detecção e Limites de Quantificação.

\begin{tabular}{|c|c|c|c|c|c|c|}
\hline Piretróides. & $\begin{array}{l}\text { Limite de } \\
\text { Detecção } \\
\left(\mathrm{mg} \mathrm{kg}^{-1}\right)\end{array}$ & $\begin{array}{c}\text { Limite de } \\
\text { Quantificação } \\
\left(\mathrm{mg} \mathrm{kg}^{-1}\right)\end{array}$ & $\begin{array}{c}\text { Nível de } \\
\text { Fortificação } \\
\left(\mathrm{mg} \mathrm{kg}^{-1}\right)\end{array}$ & $\begin{array}{c}\text { Recup. } \\
\text { Média (\%) }\end{array}$ & $\begin{array}{l}\text { Desvio } \\
\text { Padrão }\end{array}$ & $\begin{array}{c}\text { Coeficiente } \\
\text { de Variação } \\
(\%)\end{array}$ \\
\hline \multirow{3}{*}{ Fempropatrina } & \multirow{3}{*}{0,001} & \multirow{3}{*}{0,006} & 0,010 & 105 & 12,6 & 12,0 \\
\hline & & & 0,10 & 80 & 2,9 & 3,7 \\
\hline & & & 1,0 & 91 & 12,1 & 13,2 \\
\hline \multirow{3}{*}{ Fenvalerato } & \multirow{3}{*}{0,002} & \multirow{3}{*}{0,010} & 0,010 & 97 & 11,4 & 11,8 \\
\hline & & & 0,10 & 79 & 3,7 & 4,7 \\
\hline & & & 1,0 & 100 & 6,1 & 6,1 \\
\hline \multirow{3}{*}{ Cipermetrina } & \multirow{3}{*}{0,001} & \multirow{3}{*}{0,004} & 0,010 & 99 & 8,1 & 8,2 \\
\hline & & & 0,10 & 78 & 1,8 & 2,3 \\
\hline & & & 1,0 & 99 & 1,9 & 1,9 \\
\hline \multirow{3}{*}{ Deltametrina } & \multirow{3}{*}{0,002} & \multirow{3}{*}{0,008} & 0,010 & 96 & 12,2 & 12,8 \\
\hline & & & 0,10 & 72 & 2,5 & 3,5 \\
\hline & & & 1,0 & 93 & 3,5 & 3,8 \\
\hline \multirow{3}{*}{$\begin{array}{c}\text { Trans- } \\
\text { permetrina }\end{array}$} & \multirow{3}{*}{0,002} & \multirow{3}{*}{0,008} & 0,010 & 105 & 13,5 & 12,8 \\
\hline & & & 0,10 & 70 & 6,5 & 9,3 \\
\hline & & & 1,0 & 98 & 1,5 & 1,5 \\
\hline \multirow{3}{*}{ Cis-permetrina } & \multirow{3}{*}{0,002} & \multirow{3}{*}{0,010} & 0,010 & 101 & 14,5 & 14,4 \\
\hline & & & 0,10 & 80 & 10,5 & 13,1 \\
\hline & & & 1,0 & 99 & 1,7 & 1,7 \\
\hline \multirow{3}{*}{$\begin{array}{l}\text { Lambida- } \\
\text { cialotrina }\end{array}$} & \multirow{3}{*}{0,001} & \multirow{3}{*}{0,008} & 0,010 & 104 & 11,9 & 11,5 \\
\hline & & & 0,10 & 71 & 2,2 & 3,2 \\
\hline & & & 1,0 & 94 & 2,6 & 2,8 \\
\hline \multirow{3}{*}{ Bifentrina } & \multirow{3}{*}{0,002} & \multirow{3}{*}{0,010} & 0,010 & 106 & 11,0 & 10,4 \\
\hline & & & 0,10 & 85 & 5,9 & 7,0 \\
\hline & & & 1,0 & 96 & 0,9 & 0,9 \\
\hline
\end{tabular}

Os valores de coeficientes de variação porcentual estão de acordo com os índices propostos de até $20 \%$, segundo a comissão formada por membros das organizações: Association of Analytical Communities (AOAC), Food and Agriculture Organization of the United Nations (FAO), International Atomic Energy Agency (IAEA) e International Union of Pure and Applied Chemistry (IUPAC) [22]

Os limites de detecção e quantificação do método estão apresentados na Tabela 4.
Para concentrações baixas, como em análise de resíduos de pesticidas, os valores de recuperação e coeficientes de variação são geralmente semelhantes aos propostos pela comissão de especialistas de reconhecidas organizações tais como, AOAC, FAO, IAEA, EPA, IUPAC [9, 21, 22, 23]. Os CV\% apresentados na Tabelas 3 variaram entre $0,9-14,4 \%$ e estão dentro do que é comumente aceito para analise de resíduos de pesticidas, mostrando que o método possui repetitividade adequada e é preciso nas concentrações estudadas. 
A reprodutibilidade foi verificada através da realização das análises por diferentes analistas e em diferentes datas. Assim, as 7 amostras fortificadas foram separadas em dois grupos de 3 e 4 amostras. Um dos grupos foi analisado em um dia por uma equipe de analistas e o outro grupo, 5 dias depois, por outra equipe de analistas. Os valores de recuperação se mantiveram sem grandes variações nos dois grupos de amostras analisadas separadamente, com $\mathrm{CV} \%$ menores que $20 \%$, portanto estatisticamente não significativos.

\section{Resultado das análises de amostras de feijão}

Nenhuma das 48 amostras de feijão analisadas apresentou resíduos de pesticidas piretróides, resultado perfeitamente aceito visto a baixa persistência desses no meio ambiente e, também, à sua alta atividade inseticida, possibilitando sua aplicação em pequenas dosagens.

Nakagawa e Andréa [24] observaram que plântulas de feijão plantado em solo com resíduos ligados de ${ }^{14} \mathrm{C}$-atrazina, não absorveram estes resíduos. O mesmo talvez possa ocorrer com os piretróides.

Em um estudo realizado por Lopes e Dóres [25], na cidade de Aracaju, Estado de Sergipe, foi encontrado $1,83 \mathrm{mg} \mathrm{kg}^{-1}$ de deltametrina em uma amostra de feijão, juntamente com o inseticida diazinona e propanil. Nenhuma outra referência da literatura nacional descreve a presença de resíduo de piretróides em amostras de feijão. A presença destes inseticidas em amostras de feijão se dá pelo uso desses no processo de armazenamento de grãos. A aplicação de pesticidas durante o armazenamento é recomendado como a técnica com melhor custo/benefício para controle de insetos.

Os inseticidas mais recomendados para esta finalidade são os piretróides e organofosforados. Como nas amostras estudadas não se detectou a presença de piretróides, pode-se inferir a possibilidade de ocorrência de outros inseticidas, sendo que os organofosforados seriam a primeira classe a ser estudada.

\section{Conclusão}

O método desenvolvido mostrou-se eficiente para análise de feijão. Isto foi demonstrado nos resultados de validação, os quais estão de acordo com as diretrizes requisitadas para validação de métodos analíticos pelos principais órgãos nacionais e internacionais regulamentadores e normativos.
O diferencial deste método é o baixo limite de quantificação que se conseguiu em relação aos outros métodos descritos na literatura; a simplificação na determinação, devido não ter apresentado os picos dos isômeros; a necessidade de menos clean-up, quando comparado a determinação com CG-ECD.

A ausência de resíduos de piretróides nas amostras analisadas sugere a boa qualidade, em relação aos compostos analisados, do feijão consumido na cidade de São Paulo, o que atende à legislação brasileira neste setor, como ainda, indica que as normas de boas práticas agrícolas estão sendo obedecidas e seguidas. Entretanto, para que esta conclusão seja confirmada, a presença de inseticidas de outras classes, especialmente os organofosforados, precisa ser investigada.

\section{Referências}

[1] R. Hirata, Química Nova, 18(4) (1995) 368.

[2] J. Tessier, Deltametrin, Roussel-Uclaf, Paris, 1983, 25-36.

[3] J. Lhoste, Les Presses Faculte, Médecine Pharmacie de Marseille, Marseille,1966, 65p.

[4] A. B. Gebara, C. H. P. Ciscato, M. DA S. Ferreira, S. H. Monteiro, Bulletin Enviromental of Contamination and Toxicology, 75 (2005) 163.

[5] O. D. Prestes, C. A. Friggi, M. B. Adaime, R.

Zanella, Química Nova, 32(6) (2009) 1620.

[6] H. P. Vieira, A. A. Neves, M. E. L. R. Queiroz, Química Nova, 30(3) (2007) 535.

[7] Z. Chen, Y. Wang, Journal of Chromatography, A, 754 (1995) 367.

[8] H. Magalhães, Banco de Notícias, EMBRAPA Arroz e feijão, Disponível em: http://www21.sede. embrapa.br/noticias/banco de noticias/2005/janeiro/ noticia.2005-01-19.6737902350/mostra noticia, Acesso em: 05 fev. 2008.

[9] A. Ambrus, Accreditation and Quality Assurance: Journal for Quality, Comparability and Reliability in Chemical Measurement, 9(6) (2004) 288.

[10] N. M. Brito, J. O. P Amarante, L Polese, M. L. Ribeiro, Pesticidas: Resíduos - Ecotoxicologia e Meio Ambiente, 13 (2003) 129.

[11] L. Huber, LC/GC International, 11 (1998) 96.

[12] ICH - Harmonised Trapartite Guideline. Validation of analytical procedures: methodology, CPMP/ ICH/281/95, London, 1996, 9p.

[13] I. Kuselman, A. Shenhar, Analytica Chimica Acta, 
306 (1995) 301.

[14] M. E. Swartz, I. S. Krull, Pharmaceutical Technology, 2(3) (1998) 12.

[15] ANVISA, Agência Nacional de Vigilância Sanitária. Monografia de Produtos Agrotóxicos, Disponível em: http://www.anvisa.gov.br/toxicologia/monografias/ monografias.pdf. Acesso em: 15 jan. 2011.

[16] H. P. Thier, J. Kirchhoff, Manual of Pesticide Residue Analysis, Wiley: VCH, 2, 1992, 482p.

[17] H. P. Thier, H. Zeumer, Manual of Pesticide Residue Analysis, Weinheim: VCH, 1, 1987, 432p

[18] W. Specht, S. Pelz, W. Gilsbach, Fresenius Journal Analytical Chemistry, 353 (1995) 183.

[19] EPA - Environmental Protection Agency. Residue Chemistry Test Guidelines. Office of Presention, Pesticides and Toxic Substances 860.1340: Residue Analytical Method, Washington, 1996, 12p.

[20] CODEX ALIMENTARIUS. Guidelines on good laboratory practice in residue analysis, CAG/GL 401993, rev.1-2003, 36p.

[21] SANCO/10684/2009 - Method validation and quality control procedures for pesticide residues analysis in food and feed. Disponível em: www.ec.europa.eu/ food/plant/protection/resources/qualcontrol_en.pdf. Acesso em 06 nov. 2010.

[22] AOAC/FAO/IAEA/IUPAC - Expert Consultation; Guidelines for Single-laboratory Validation of Analytical Methods for Trace-level Conc. Of Organic Chemicals. Miskolc. Hungary, 1999.

[23] J.M. Green, Analytical Chemistry News \& Features, 68(9) (1996) 305A.

[24] L. E. Nakagawa, M. M. Andréa, Pesquisa Agropecuária Brasileira, 35(8) (2000) 1517.

[25] W.G. Lopes, H. G. Dóres, Pesticidas: Resíduos Ecotoxicologia e Meio Ambiente, 13 (2003) 13. 